\title{
DELAY DIFFERENTIAL EQUATIONS OF ODD ORDER SATISFYING PROPERTY $P_{k}$
}

\author{
RAYMOND D. TERRY
}

(Received 16 October 1974)

\begin{abstract}
The property $P_{k}(k=0,1, \cdots, n)$ is formulated. For $k=0, n$ this property reduces to conditions $A$ and $B$ defined by Kiguradze (1962) for a class of ordinary differential equations. Sufficient conditions are then given which guarantee that a class of delay differential equations of

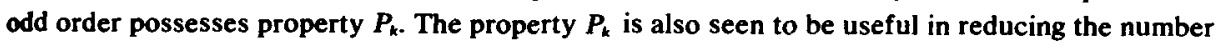
of types of positive solutions of a related nonhomogeneous delay differential equation.
\end{abstract}

The equation

$$
D^{m} y(t)+F[t, y(t)]=0, m \geqq 2
$$

has been considered by various authors subject to additional sign and monotone properties on $F(t, u)$. Briefly, a solution of (1) or of (2) below is called oscillatory on $[a, \infty)$ if for each $\alpha>a$ there is a $\beta>\alpha$ such that $y(\beta)=0$. It is called nonoscillatory otherwise. Paralleling the development by Kiguradze (1962) we adopt the following terminology.

Definition 1. For $m=2 n+1$, a positive solution y of (1) is of type $A_{k}$ $(k=0, \cdots, n)$ if for $t$ sufficiently large $D^{j} y(t)>0$ for $j=0, \cdots, 2 k$ and $(-1)^{j} D^{j} y(t)>0$ for $j=2 k+1, \cdots, 2 n$.

Definition 2. For $m=2 n$, a positive solution $y$ of (1) is of type $A_{k}$ $(k=0, \cdots, n-1)$ if for $t$ sufficiently large $D^{j} y(t)>0$ for $j=0, \cdots, 2 k+1$ and $(-1)^{j+1} D^{j} y(t)>0$ for $j=2 k+2, \cdots, 2 n-1$.

Definition 3. Equation (1) is said to satisfy condition $A$ if (1) has an oscillatory solution and every nonoscillatory solution tends to zero monotonically as $t \rightarrow \infty$.

DefinItIon 4. Equation (1) satisfies condition $B$ if a solution $y$ is either oscillatory or $\lim _{t \rightarrow \infty} D^{m-1} y(t)=0$. 
It has been shown in Kiguradze (1962) that a positive solution of (1) is necessarily of type $A_{k}$ for some admissible $k$. For $m=2 n$, (1) fulfills condition $A$ if, and only if, all solutions are oscillatory. For $m=2 n+1$, (1) satisfies condition $A$ if, and only if, there are no solutions of type $A_{r}(r=1, \cdots, n)$ and every solution of type $A_{0}$ tends to zero monotonically as $t \rightarrow \infty$.

In section one we consider a homogeneous delay differential equation of odd order and formulate a property $P_{k}$ which includes both conditions $A$ and $B$ as special cases. Section two is devoted to providing sufficient conditions for the equation to possess property $\boldsymbol{P}_{k}$. In section three an a priori classification according to types $C_{k}^{R}$ is introduced for the positive solutions of a nonhomogeneous delay differential equation of odd order. The property $P_{k}$ is seen to be useful in reducing the kinds of positive solutions admissible.

\section{1}

In this section and the next we shall consider the homogeneous delay differential equation

$$
D^{2 n+1-i}\left[r(t) D^{i} y(t)\right]+y_{\tau}(t) f\left[t, y_{\tau}(t)\right]=0,
$$

where $0<m \leqq r(t) \leqq M<\infty, 0 \leqq \tau(t) \leqq T<\infty, y_{\tau}(t)=y[t-\tau(t)]$ and $f(t, u)$ satisfies the following properties:

$$
f(t, u) \text { is a continuous real-valued function on }[0, \infty) \times R ;
$$

$$
\text { for each fixed } t \in[0, \infty), f(t, u)<f(t, v) \text { for } 0<u<v \text {; and }
$$

(F3) for each fixed $t \in[0, \infty), f(t, u)>0$ and $f(t,-u)=f(t, u)$ for $u \neq 0$.

We first let

$$
y_{j}(t)= \begin{cases}D^{j} y(t), & j=0, \cdots, i-1 \\ D^{j-i}\left[r(t) D^{i} y(t)\right], & j=i, \cdots, 2 n .\end{cases}
$$

Analogous to Definition 1 we shall classify the positive solutions of (2).

Definition 5. A positive solution y of (2) is of type $C_{k}$ on $\left[T_{0}, \infty\right)$ if for $t \geqq T_{0} y_{j}(t)>0(j=0, \cdots, 2 k)$ and $(-1)^{j} y_{j}(t)>0(j=2 k+1, \cdots, 2 n)$.

As in Terry $(1973,1974)$, it is evident that a positive solution of (2) is necessarily of type $C_{k}$ for some $k=0, \cdots, n$. Moreover, the following two lemmas may be established.

Lemma 1. Let y be a solution of (2) of type $C_{k}, k \geqq 1$. Then there exist numbers $N_{j}^{k}>0(j=0, \cdots, 2 k)$ such that

$$
\begin{aligned}
\left(t-T_{1}\right) y_{j}(t) & \leqq N_{i}^{k} y_{j-1}(t), t \geqq T_{1}=T_{0}+T \text { and } \\
t y_{j}(t) & \leqq 2 N_{j}^{k} y_{j-1}(t), \quad t \geqq 2 T_{1} .
\end{aligned}
$$


Lemma 2. Let y be a solution of (2) of type $C_{k}, k \geqq 1$. Then there exist numbers $k_{i}>0$ and $t_{j} \geqq T_{1}(j=0, \cdots, 2 k-1)$ such that

$$
y_{j r}(t)=y_{j}(t-\tau(t)) \geqq k_{j} y_{j}(t), \quad t \geqq t_{j} .
$$

While it is of interest to obtain specific estimates for the numbers $N_{j}^{k}$, this is unnecessary for the subsequent development of this paper. As in Terry (1973), these two lemmas and the later results may be extended to the case where $\tau(t)$ satisfies either of the two conditions

$$
\begin{aligned}
& 0 \leqq \tau(t) \leqq \mu t, \quad 0 \leqq \mu<m /(m+M), \text { or } \\
& 0 \leqq \tau(t) \leqq \mu t^{\beta}, \quad 0 \leqq \mu<\infty \text { and } 0 \leqq \beta<1,
\end{aligned}
$$

provided $T_{1}$ is reinterpreted as $\min \left\{t>T_{0}: t-\tau(t) \geqq T_{0}\right.$ for $\left.t \geqq T_{1}\right\}$.

DEFINITION 6. Equation (2) fulfills property $P_{k}$ if, and only if, (2) has no solutions of types $C_{r}(r=k+1, \cdots, n)$ and for any solution $y(t)$ of type $C_{k}$ the intermediate function $y_{2 k}(t)$ tends to zero monotonically as $t \rightarrow \infty$.

When $r \equiv 1$ and $\tau \equiv 0$, the classification of solutions of (2) according to types $C_{k}$ coincides with that of Kiguradze (1962). Moreover, property $P_{0}$ is the natural analogue of condition $A$; property $P_{n}$ corresponds to condition $B$.

2

We now seek to prescribe conditions which ensure that equation (2) fulfills the property $P_{k}$.

Theorem 1. Let y be a positive solution of (2) of type $C_{k}$. Then $y_{2 k}(t)$ tends to zero monotonically as $t \rightarrow \infty$ if for all positive constants $C$

$$
\int^{\infty} t^{2 n} f\left(t, C t^{2 k}\right) d t=+\infty
$$

Proof. Let $y$ be a solution of (2) of type $C_{k}$ on $\left[T_{0}, \infty\right)$. Then for $t \geqq T_{1}$, $y_{\tau}(t)>0, y_{j}(t)>0(j=0, \cdots, 2 k)$ and $(-1)^{j} y_{j}(t)>0(j=2 k+1, \cdots, 2 n)$. Multiplying (2) by $t^{2 n-2 k}$ and integrating from $T_{1}$ to $t \geqq T_{1}$

$$
\int_{T_{1}}^{t} s^{2 n-2 k} D y_{2 n}(s) d s+\int_{T_{1}}^{t} s^{2 n-2 k} y_{\tau}(s) f\left[s, y_{\tau}(s)\right] d s=0 .
$$

Integrating the first term by parts

$$
\begin{aligned}
I & \equiv \int_{T_{1}}^{t} s^{2 n-2 k} D y_{2 n}(s) d s=\left[s^{2 n-2 k} y_{2 n}(s)\right]_{T_{\mathrm{t}}}^{t} \\
& -(2 n-2 k) \int_{T_{1}}^{t} s^{2 n-2 k-1} y_{2 n}(s) d s
\end{aligned}
$$


An easy induction yields

$$
I=\left[\sum_{j=0}^{l}(-1)^{j}(2 n-2 k)_{j} s^{2 n-2 k-i} y_{2 n-j}(s)\right] T_{1}
$$

$$
+(-1)^{l+1}(2 n-2 k)_{t+1} \int_{T_{1}}^{t} s^{2 n-2 k-t-1} y_{2 n-l}(s) d s,
$$

where $0 \leqq j \leqq l \leqq 2 n-i,(n)_{0}=1$ and $(n)_{k}=n \cdots(n-k+1)$ for $k \geqq 1$. If $2 k \geqq i, 2 n-2 k \leqq 2 n-i$ and we may let $l=2 n-2 k-1$ in (4) to obtain

$$
\begin{aligned}
I & \left.=\left[\sum_{j=0}^{2 n-2 k-1}(-1)^{i}(2 n-2 k)_{j} s^{2 n-2 k-j} y_{2 n-j}(s)\right]\right]_{T_{1}} \\
& +(-1)^{2 n-2 k}(2 n-2 k)_{2 n-2 k} \int_{T_{1}}^{t} y_{2 k+1}(s) d s .
\end{aligned}
$$

On the other hand, if $1<2 k<i$, then $2 n-2 k>2 n-i$. We let $l=2 n-i$ in (4) and observe that

$$
\begin{aligned}
(-1)^{l+1} y_{2 n-1}(s) & =(-1)^{2 n-i+1} y_{i}(s)=(-1)^{i+1} y_{i}(s) \\
& =(-1)^{i+1} r(s) D^{i} y(s) \\
& \geqq(-1)^{i+1} M D^{i} y(s) .
\end{aligned}
$$

Then (4) becomes

$$
I \geqq\left[\sum_{j=0}^{2 n-i}(-1)^{j}(2 n-2 k)_{j} s^{2 n-2 k-j} y_{2 n-j}(s)\right]_{T_{1}}
$$

$$
+(-1)^{2 n-i+1} M(2 n-2 k)_{2 n-i+1} \int_{T}^{t} s^{i-2 k-1} D y_{i-1}(s) d s .
$$

We now examine the latter integral. An integration by parts results in

$$
\begin{aligned}
J \equiv \int_{T_{1}}^{t} s^{i-2 k-1} D y_{i-1}(s) d s & =\left[s^{i-2 k-1} y_{i-1}(s)\right]_{T_{1}}^{t} \\
& -(i-2 k-1) \int_{T_{1}}^{t} s^{i-2 k-2} y_{i-1}(s) d s .
\end{aligned}
$$

This serves as the anchor for another inductive argument based on further integration by parts. It follows that

$$
\begin{aligned}
J & =\left[\sum_{j=1}^{L}(-1)^{j+1}(i-2 k-1)_{j-1} s^{i-2 k-j} y_{i-j}(s)\right]_{T_{1}} \\
& +(-1)^{L}(i-2 k-1)_{L} \int_{T_{1}}^{t} s^{i-2 k-j-1} y_{i-L}(s) d s,
\end{aligned}
$$


where $1 \leqq j \leqq L \leqq i-2 k-1$. Combining (6) and (7), we obtain

$$
\begin{aligned}
I \geqq \sum_{j=0}^{2 n-i}(-1)^{j}(2 n-2 k)_{j} s^{2 n-2 k-j} y_{2 n-j}(s) \\
\left.+N_{1} \sum_{j=1}^{L}(-1)^{j+1}(i-2 k-1)_{j} s^{i-2 k-j} y_{i-j}(s)\right] T_{i} \\
+N_{1} N_{2} \int_{T_{1}}^{t} y_{i-L}(s) d s,
\end{aligned}
$$

where $N_{1}=(-1)^{i+1} M(2 n-2 k)_{2 n-i+1}$ and $N_{2}=(-1)^{L}(i-2 k-1)_{L}$. We may let $L=i-2 k-1$ in this and observe that

$$
\operatorname{sgn} N_{1} N_{2}=(-1)^{i+1}(-1)^{L}=(-1)^{i+1}(-1)^{i-2 k-1}=+1 .
$$

It follows that

(8a)

$$
\begin{aligned}
{\left[F_{2 k}(s)\right]_{T_{1}}^{t} } & +(2 n-2 k) ! \int_{T_{1}}^{t} y_{2 k+1}(s) d s \\
& +\int_{T_{1}}^{l} s^{2 n-2 k} y_{\tau}(s) f\left[s, y_{\tau}(s)\right] d s=0
\end{aligned}
$$

for $i \leqq 2 k$ and

(8b)

$$
\left[\bar{F}_{2 k}(s)\right]_{T_{1}}^{t}+N_{1} N_{2} \int_{T_{1}}^{t} y_{2 k+1}(s) d s
$$

$$
+\int_{T_{1}}^{t} s^{2 n-2 k} y_{\tau}(s) f\left[s, y_{\tau}(s)\right] d s \leqq 0
$$

for $i>2 k$, where

$$
\begin{aligned}
F_{2 k}(s)= & \sum_{j=0}^{2 n-2 k-1}(-1)^{j}(2 n-2 k)_{j} s^{2 n-2 k-j} y_{2 n-j}(s) \text { and } \\
\bar{F}_{2 k}(s)= & \sum_{j=0}^{2 n-i}(-1)^{i}(2 n-2 k)_{j} s^{2 n-2 k-j} y_{2 n-j}(s) \\
& +N_{1} \sum_{j=1}^{i-2 k-1}(-1)^{i}(i-2 k-1)_{j} s^{i-2 k-j} y_{i-j}(s)
\end{aligned}
$$

We note that each term of $F_{2 k}(s)$ is positive on $\left[T_{1}, \infty\right)$ since

$$
(-1)^{j} y_{2 n-j}(s)=(-1)^{2 n-j}(s)=(-1)^{p} y_{p}(s)
$$

and $p=2 n-j \geqq 2 k+1$ since $0 \leqq j \leqq 2 n-2 k-1$. Similarly, each term of $\bar{F}_{2 k}(s)$ is positive since

$$
(-1)^{i+1}(-1)^{i+1} y_{i-j}(s)=(-1)^{i-j} y_{i-j}(s)=(-1)^{q} y_{q}(s)
$$

and $q=i-j \geqq 2 k+1$ since $j \leqq i-2 k-1$. Moreover, 


$$
\begin{aligned}
\int_{T_{1}}^{t} y_{2 k+1}(s) d s & =y_{2 k}(t)-y_{2 k}\left(T_{1}\right) \quad \text { if } \quad 2 k+1 \neq i \\
& =\int_{T_{1}}^{t} r(s) D^{i} y(s) \quad \text { if } \quad 2 k+1=i \\
& \geqq M \int_{T_{1}}^{t} D^{i} y(s)=M\left[\dot{y}_{2 k}(t)-y_{2 k}\left(T_{1}\right)\right]
\end{aligned}
$$

Let us assume that $\lim _{t \rightarrow \infty} y_{2 k}(t)=\gamma>0$. Since $y_{2 k+1}(t)<0$ on $\left[T_{1}, \infty\right), y_{2 k}(t)$ is a decreasing function of $t$ on $\left[T_{1}, \infty\right)$ and $y_{2 k}(t) \geqq \gamma, t \geqq T_{1}$. By Lemma 1

$$
y(s) \geqq N s^{2 k} y_{2 k}(s) \geqq N \gamma s^{2 k}, \text { where } N^{-1}=N_{1}^{k} N_{2}^{k} \cdots N_{2 k}^{k} \text {. }
$$

Hence,

$$
y_{\tau}(s) \geqq N \gamma[s-\tau(s)]^{2 k} \geqq N \gamma(1-\mu)^{2 k} s^{2 k}
$$

if $\tau$ satisfies (T1). On the other hand, if $\tau$ satisfies (T2), there is a $T_{2} \geqq T_{1}$ such that $s-\tau(s) \geqq s / 2$ for $s \geqq T_{2}$, which implies that $y_{\tau}(s) \geqq N \gamma 2^{-2 k} s^{2 k}$ for $s \geqq T_{2}$. As we may replace $T_{1}$ by $T_{2}$ in the above considerations, we may assume, without loss of generality, that $T_{2}=T_{1}$. Thus, in either case $y_{\tau}(s) \geqq C s^{2 k}$ on the appropriate interval so that

$$
\begin{aligned}
s^{2 n-2 k} y_{\tau}(s) f\left[s, y_{\tau}(s)\right] & \geqq s^{2 n-2 k} C s^{2 k} f\left[s, C s^{2 k}\right] \\
& =C s^{2 n} f\left(s, C s^{2 k}\right) .
\end{aligned}
$$

For $i \leqq 2 k$ we substitute in (8a)

$$
\left[F_{2 k}(s)\right]_{T_{1}}^{s}+(2 n-2 k) !\left[y_{2 k}(s)\right]_{T_{1}}^{t} s^{2 n} f\left(s, C s^{2 k}\right) d s \leqq 0 .
$$

Transposing,

$$
\int_{T_{1}}^{t} s^{2 n} f\left(s, C s^{2 k}\right) d s \leqq C^{-1}\left[F_{2 k}\left(T_{1}\right)+(2 n-2 k) ! y_{2 k}(t)\right] .
$$

This contradicts (3) in the case $\tau$ satisfies (T1); if $\tau$ satisfies (T2), we replace $T_{1}$ by $T_{2}$ and obtain the same contradiction. For $i>2 k$, we substitute in (8b) instead.

THEOREM 2. Let $\phi$ be a function satisfying $\phi(y)>0, \phi^{\prime}(y) \geqq 0$ and

$$
\int^{\infty} \frac{d y}{y \phi(y)}<\infty
$$

then (2) fulfills property $P_{k}$ if for all positive constants $C$

$$
\int^{\infty} t^{2 n} f\left(t, C t^{2 k}\right) \phi^{-1}(t) d t=+\infty
$$


Proof. Suppose that $y$ is a solution of (2) of type $C_{k}$ on $\left[T_{0}, \infty\right)$, where $k \geqq 1$. Then multiplying equation (2) by $t^{2 n}[\phi(t) y(t)]^{-1}$ and integrating from $T_{1}$ to $t>T_{1}$

$$
\int_{T_{1}}^{t} s^{2 n} D y_{2 n}(s)[\phi(s) y(s)]^{-1} d s+\int_{T_{1}}^{t} s^{2 n} \frac{f\left[s, y_{\tau}(s)\right.}{\phi(s) y(s)} d s=0 .
$$

We denote the first integral by $I_{1}$. An integration by parts yields

$$
I_{1}=\left[\gamma(s)(\phi(s) y(s))^{-1}\right]_{T_{1}}^{t}-\int_{T_{1}}^{t} \gamma(s) D\left((\phi(s) y(s))^{-1}\right) d s,
$$

where $\gamma(s)=D^{-1}\left(s^{2 n} D y_{2 n}(s)\right)$. Specifically, one integration by parts gives

$$
\gamma(s)=s^{2 n} y_{2 n}(s)-2 n D^{-1}\left(s^{2 n-1} y_{2 n}(s)\right) .
$$

We may establish by induction that

$$
\begin{aligned}
\gamma(s) & =\sum_{j=0}^{p}(-1)^{i}(2 n)_{j} s^{2 n-j} y_{2 n-j}(s) \\
& +\left(-1^{p+1}(2 n)_{p+1} D^{-1}\left[s^{2 n-p-1} y_{2 n-p}(s)\right]\right.
\end{aligned}
$$

for $0 \leqq j \leqq p \leqq 2 n-i$. If $2 k \geqq i, 2 n-2 k \leqq 2 n-i$ and we may let $p=2 n-2 k$ in (12) to obtain

$$
\gamma(s)=\sum_{j=0}^{2 n-2 k}(-1)^{i}(2 n)_{j} s^{2 n-i} y_{2 n-j}(s)+N_{0} D^{-1}\left[s^{2 k-1} y_{2 k}(s)\right],
$$

where $N_{0}=(-1)^{2 n-2 k+1}(2 n)_{2 n-2 k+1}$. We define $\gamma_{0}(s)$ by

$$
\gamma(s)=\gamma_{0}(s)+N_{0} D^{-1}\left[s^{2 k-1} y_{2 k}(s)\right] .
$$

Then, a substitution in (11) produces

$$
\begin{aligned}
I_{1}=\left[\left(\gamma_{0}(s)\right.\right. & \left.\left.+N_{0} D^{-1}\left(s^{2 k-1} y_{2 k}(s)\right)\right)(\phi(s) y(s))^{-1}\right]_{T_{1}}^{t} \\
& -\int_{T_{1}}^{t}\left(\gamma_{0}(s)+N_{0} D^{-1}\left(s^{2 k-1} y_{2 k}(s)\right) D\left((\phi(s) y(s))^{-1}\right) d s\right. \\
=\left[\gamma_{0}(s)\right. & \left.(\phi(s) y(s))^{-1}\right]_{T_{1}}^{t}-\int_{T_{1}}^{t} \gamma_{0}(s) D\left((\phi(s) y(s))^{-1}\right) d s \\
& +N_{0}\left[D^{-1}\left(s^{2 k-1} y_{2 k}(s)\right) / \phi(s) y(s)\right]_{T_{1}}^{t} \\
& -N_{0} \int_{T_{1}}^{t} D^{-1}\left(s^{2 k-1} y_{2 k}(s)\right) D\left((\phi(s) y(s))^{-1}\right) d s .
\end{aligned}
$$

Applying the integration-by-parts formula in reverse, we recombine the last two terms to obtain 


$$
\begin{aligned}
I_{1}=\left[\gamma_{0}(s)(\phi(s) y(s))^{-1}\right]_{T_{1}}^{t} & -\int_{T_{1}}^{t} \gamma_{0}(s) D\left((\phi(s) y(s))^{-1}\right) d s \\
& +N_{0} \int_{T_{1}}^{t} \frac{s^{2 k-1} y_{2 k}(s)}{\phi(s) y(s)} d s .
\end{aligned}
$$

By Lemma 1 there is a number $N_{2 k}=N_{1}^{k} \cdots N_{2 k}^{k}>0$ such that

$$
t^{2 k} y_{2 k}(t) \leqq N_{2 k} y(t) \text { for } t \geqq 2 T_{1} \text {. }
$$

Thus

$$
\frac{s^{2 k-1} y_{2 k}(s)}{\phi(s) y(s)}=\frac{s^{2 k} y_{2 k}(s)}{s \phi(s) y(s)} \leqq \frac{N_{2 k}}{s \phi(s)} .
$$

Since $N_{0}=-\left|N_{0}\right|<0$,

$$
\begin{gathered}
I_{1} \geqq\left[\gamma_{0}(s)(\phi(s) y(s))^{-1}\right]_{T_{1}}^{t}-\int_{T_{1}}^{t} \gamma_{0}(s) D\left((\phi(s) y(s))^{-1}\right) d s \\
-\left|N_{0}\right| N_{2 k} \int_{2 T_{1}}^{t} \frac{d s}{s \phi(s)} .
\end{gathered}
$$

Otherwise, if $2 k<i$, we continue the inductive procedure defined by (12) until $p=2 n-i$. Then (12) becomes

$$
\gamma(s)=\sum_{i=0}^{2 n-i}(-1)^{i}(2 n)_{j} s^{2 n-j} y_{2 n-j}(s)+N_{1} D^{-1}\left[s^{i-1} y_{i}(s)\right],
$$

where $N_{1}=(-1)^{2 n-i+1}(2 n)_{2 n-i+1}$. Letting

$$
\gamma(s)=\gamma_{1}(s)+N_{1} D^{-1}\left[s^{i-1} y_{i}(s)\right]
$$

it follows as before upon substitution in (11) that

$$
\begin{aligned}
I_{1}=\left[\gamma_{1}(s)(\phi(s) y(s))^{-1}\right]_{T_{1}}^{\prime} & -\int_{T_{1}}^{1} \gamma_{1}(s) D\left((\phi(s) y(s))^{-1}\right) d s \\
& +N_{1} \int_{T_{1}}^{1} \frac{s^{i-1} y_{i}(s)}{\phi(s) y(s)} d s .
\end{aligned}
$$

We observe that

$$
\begin{aligned}
N_{1} y_{i}(s) & =\left|N_{1}\right|(-1)^{2 n-i+1} y_{i}(s)=\left|N_{1}\right|(-1)^{i+1} y_{i}(s) \\
& =\left|N_{1}\right|(-1)^{i+1} r(s) D y_{i-1}(s) \\
& \geqq\left|N_{1}\right|(-1)^{i+1} M D y_{i-1}(s)=M N_{1} D y_{i-1}(s) .
\end{aligned}
$$

Thus,

$$
\begin{aligned}
I_{1} \geqq\left[\gamma_{1}(s)(\phi(s) y(s))^{-1}\right]_{T_{1}}^{t}-\int_{T_{t}}^{1} \gamma_{1}(s) D\left((\phi(s) y(s))^{-1}\right) d s \\
+N_{1} M \int_{T_{1}}^{1} \frac{s^{i-1} D y_{i-1}(s)}{\phi(s) y(s)} d s .
\end{aligned}
$$


Analogous to (11) we have

$$
\begin{aligned}
I_{2}=\int_{T_{1}}^{t} \frac{s^{i-1} D y_{i-1}(s)}{\phi(s) y(s)} d s & =\left[\gamma_{2}(s)(\phi(s) y(s))^{-1}\right]_{T_{1}}^{t} \\
& -\int_{T_{1}}^{t} \gamma_{2}(s) D\left((\phi(s) y(s))^{-1}\right) d s,
\end{aligned}
$$

where $\gamma_{2}(s)=D^{-1}\left[s^{i-1} D y_{i-1}(s)\right]$. We find upon one integration that

$$
\gamma_{2}(s)=s^{i-1} y_{i-1}(s)-(i-1) D^{-1}\left[s^{i-2} y_{i-1}(s)\right] .
$$

An inductive argument yields

$$
\begin{aligned}
\gamma_{2}(s)= & \sum_{j=1}^{p}(-1)^{i+1}(i-1)_{j-1} s^{i-j} y_{i-i}(s) \\
& +(-1)^{p}(i-1)_{p} D^{-1}\left[s^{i-p-1} y_{i-p}(s)\right],
\end{aligned}
$$

for $i \leqq j \leqq p \leqq i-2 k$. Letting $p=i-2 k$ results in

where

$$
\begin{aligned}
\gamma_{2}(s)= & \sum_{j=1}^{i-2 k}(-1)^{i+1}(i-1)_{j-1} s^{i-j} y_{i-j}(s) \\
& +(-1)^{i-2 k}(i-1)_{i-2 k} D^{-1}\left[s^{2 k-1} y_{2 k}(s)\right] \\
= & \gamma_{3}(s)+N_{2} D^{-1}\left[s^{2 k-1} y_{2 k}(s)\right],
\end{aligned}
$$

$$
\gamma_{3}(s)=\sum_{j=1}^{i-2 k}(-1)^{j+1}(i-1)_{j-1} s^{i-j} y_{i-j}(s)
$$

and

$$
N_{2}=(-1)^{i-2 k}(i-1)_{i-2 k} .
$$

To simplify the expressions involved, let

$$
\begin{aligned}
\Gamma(s) & =\gamma_{1}(s)+M N_{1} \gamma_{2}(s) \\
& =\gamma_{1}(s)+M N_{1}\left[\gamma_{3}(s)+N_{2} D^{-1}\left(s^{2 k-1} y_{2 k}(s)\right)\right] \\
& =\gamma_{1}(s)+M N_{1} \gamma_{3}(s)+M N_{1} N_{2} D^{-1}\left[s^{2 k-1} y_{2 k}(s)\right] \\
& =\Gamma_{0}(s)+M N_{1} N_{2} D^{-1}\left[s^{2 k-1} y_{2 k}(s)\right] \\
& =\Gamma_{0}(s)-M\left|N_{1} N_{2}\right| D^{-1}\left[s^{2 k-1} y_{2 k}(s)\right] .
\end{aligned}
$$

We note here that $\operatorname{sgn} N_{1} N_{2}=(-1)^{2 n-i+1}(-1)^{i-2 k}=(-1)^{1}=-1$. Substituting in (14),

$$
\begin{aligned}
I_{1} \geqq & {\left[\gamma_{1}(s)(\phi(s) y(s))^{-1}\right]_{T_{1}}^{t}-\int_{T_{1}}^{t} \gamma_{1}(s) D\left((\phi(s) y(s))^{-1}\right) d s } \\
& +M N_{1}\left[\left(\gamma_{3}(s)+N_{2} D^{-1}\left(s^{2 k-1} y_{2 k}(s)\right)\right) / \phi(s) y(s)\right]_{T_{1}}^{\prime} \\
& -M N_{1} \int_{T_{1}}^{l}\left(\gamma_{3}(s)+N_{2} D^{-1}\left(s^{2 k-1} y_{2 k}(s)\right)\right) D\left((\phi(s) y(s))^{-1}\right) d s
\end{aligned}
$$




$$
\begin{aligned}
= & {\left[\Gamma_{0}(s)(\phi(s) y(s))^{-1}\right]_{T_{1}}^{t}-\int_{T_{1}}^{t} \Gamma_{0}(s) D\left((\phi(s) y(s))^{-1}\right) d s } \\
& +M N_{1} N_{2} \int_{T_{1}}^{t} \frac{s^{2 k-1} y_{2 k}(s)}{\phi(s) y(s)} d s .
\end{aligned}
$$

As in the discussion preceding (13) we conclude that

$$
\begin{gathered}
I_{1} \tau\left[\Gamma_{0}(s)(\phi(s) y(s))^{-1}\right]_{T_{1}}^{t}-\int_{T_{1}}^{t} \Gamma_{0}(s) D\left((\phi(s) y(s))^{-1}\right) d s \\
-M\left|N_{1} N_{2}\right| \int_{2 T_{1}}^{t} \frac{d s}{s \phi(s)} .
\end{gathered}
$$

We next consider the second integral in (10). By Lemma 2 there is a $k_{0}>0$ and a $t_{0}>T_{1}$ such that $y_{\tau}(s) \geqq k_{0} y(s)$ for $s \geqq t_{0}$. Since $y_{2 k}(s)>0, y_{2 k-1}(s)$ is increasing on $\left[T_{0}, \infty\right)$ and there is a $C_{0}>0$ such that $y_{2 k-1}(s) \geqq C_{0}$ for $s \geqq T_{1}$. Moreover, by Lemma 1 there is an $N_{2 k-1}=N_{1}^{k} \cdots N_{2 k-1}^{k}$ such that $s^{2 k-1} y_{2 k-1}(s) \leqq N_{2 k-1} y(s)$ for $s \geqq 2 T_{1}$. Thus,

$$
y_{\tau}(s) \geqq k_{0} y(s) \geqq k_{0} N_{2 k-1}^{-1} s^{2 k-1} y_{2 k-1}(s) \geqq k_{0} N_{2 k-1}^{-1} C_{0} s^{2 k-1} .
$$

By (iii)

$$
s^{2 n} f\left[s, y_{\tau}(s)\right] y_{\tau}(s) y^{-1}(s) \phi^{-1}(s) \geqq k_{0} s^{2 n} f\left(s, C_{1} s^{2 k-1}\right) \phi^{-1}(s),
$$

where $C_{1}=k_{0} N_{2 k-1}^{-1} C_{0}$ and $s \geqq T_{*}=\max \left\{t_{0}, 2 T_{1}\right\}$. As a result,

$$
\begin{aligned}
\int_{T_{1}}^{t} \frac{s^{2 n} f\left[s, y_{\tau}(s)\right] y_{\tau}(s) d s}{\phi(s) y(s)} & \geqq \int_{T_{*}}^{t} \frac{s^{2 n} f\left[s, y_{\tau}(s)\right] y_{\tau}(s) d s}{\phi(s) y(s)} \\
& \geqq k_{0} \int_{T_{*}}^{t} s^{2 n} f\left(s, C_{1} s^{2 k-1}\right) d s / \phi(s) .
\end{aligned}
$$

Substituting (16) together with (13) or (15) in (10), we obtain

$\left[\gamma_{0}(s)(\phi(s) y(s))^{-1}\right]_{T_{1}}^{t}-\int_{T_{1}}^{t} \gamma_{0}(s) D\left((\phi(s) y(s))^{-1}\right) d s$

(17a)

$$
-\left|N_{0}\right| N_{2 k} \int_{2 T_{1}}^{t} \frac{d s}{s \phi(s)}+k_{0} \int_{T_{*}}^{t} s^{2 n} f\left(s, C_{1} s^{2 k-1}\right) d s / \phi(s) \leqq 0 \quad \text { for } 2 k \geqq i
$$

or

$\left[\Gamma_{0}(s)(\phi(s) y(s))^{-1}\right]_{T_{1}}^{t}-\int_{T_{1}}^{t} \Gamma_{0}(s) D\left((\phi(s) y(s))^{-1}\right) d s$

(17b)

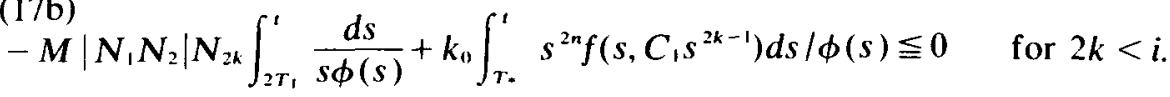


We note that each term of $\gamma_{0}(s)$ or of $\Gamma_{0}(s)$ is positive on $\left[T_{1}, \infty\right)$. Since $k \geqq 1, y^{\prime}(s) \geqq 0$ and

$$
-D\left((\phi(s) y(s))^{-1}\right)=\frac{D(\phi(s) y(s))}{[\phi(s) y(s)]^{2}}=\frac{\phi(s) y^{\prime}(s)+\phi^{\prime}(s) y(s)}{[\phi(s) y(s)]^{2}}>0 .
$$

Consequently,

$$
\int_{T}^{t} s^{2 n} f\left(s, C_{1} s^{2 k-1}\right) \phi^{-1}(s) d s \leqq\left\{\begin{array}{l}
k_{0}^{-1}\left[\gamma_{0}(\phi y)\left(T_{1}\right)+\left|N_{0}\right| N_{2 k} \int_{2 T_{1}}^{t} \frac{d s}{s \phi(s)}\right] \\
k_{0}^{-1}\left[\Gamma_{0}(\phi y)\left(T_{1}\right)+M\left|N_{1} N_{2}\right| N_{2 k} \int_{2 T_{1}}^{t} \frac{d s}{s \phi(s)}\right] .
\end{array}\right.
$$

Thus the condition

$$
\int^{x} t^{2 n} f\left(t, C t^{2 k-1}\right) \phi^{-1}(t) d t=\infty, \quad k \geqq 1
$$

will imply that (2) has no $C_{r}$-solutions $(r=k, \cdots, n)$, that is the condition

$$
\int^{\infty} t^{2 n} f\left(t, C t^{2 k+1}\right) \phi^{-1}(t) d t=\infty, \quad k \geqq 0
$$

will imply that (2) has no $C_{r}$-solution $r=k+1, \cdots, n$ ). A fortiori, (9b) implies that (2) has no $C_{r}$-solutions $(r=k+1, \cdots, n)$, where $k \geqq 0$. In addition, the conditions $\phi>0$ and $\phi^{\prime} \geqq 0$ show that there is a $k>0$ such that $\phi(t) \geqq k$ so that

$$
\begin{aligned}
\int^{\infty} t^{2 n} f\left(t, C t^{2 k}\right) d t & =k\left[\frac{1}{k} \int^{\infty} t^{2 n} f\left(t, C t^{2 k}\right) d t\right] \\
& \geqq k \int^{\infty} t^{2 n} f\left(t, C t^{2 k}\right) \phi^{-1}(t) d t .
\end{aligned}
$$

Thus, the integral condition of (9b) implies that of (3). By Theorem 1, any $C_{k}$-solution $y$ of (2) will satisfy $\lim _{t \rightarrow \infty} y_{2 k}(t)=0$. It follows that (2) possesses property $P_{k}$.

By modifying the conditions on $f$ and $\phi$, we may obtain a simpler criterion for the presence of property $P_{k}$.

DEFINITION 7. The function $f$ is nonlinear with strength coefficient $2 n+1-j$ $(j=0, \cdots, 2 n+1)$ if, and only if, there is a function $\phi$ satisfying $\phi(u)>0$, $\phi^{\prime}(u) \geq 0$,

$$
\int^{\infty} \frac{d u}{u \phi(u)}<\infty
$$

and $f(t, u) \geqq \phi(u) f\left(t, C t^{j}\right)$. 
When $j=0$, the strength coefficient is maximal and $f(t, u)$ is called strongly nonlinear.

In the presence of some degree of nonlinearity, the hypotheses of Theorem 2 may be proportionately weakened. Suppose that $f$ is nonlinear with strength coefficient $2 n+1-2 j$ and $\phi^{\prime \prime}(u)<0$. Then, multiplying (2) by $t^{2 n}[\phi(y) y]^{-1}$, we obtain as in the proof of Theorem 2

$$
\begin{aligned}
& {\left[\gamma_{0}(s)(y(s) \phi(y(s)))^{-1}\right]_{T_{1}}^{t}-\int_{T_{1}}^{t} \gamma_{0}(s) D\left((y(s) \phi(y(s)))^{-1}\right) d s} \\
& \quad-\int_{T_{1}}^{t} \frac{s^{2 k-1} y_{2 k}(s)}{y(s) \phi(y(s))} d s+\int_{T_{1}}^{t} s^{2 n} y_{\tau}(s) f\left[s, y_{\tau}(s)\right] d s / \phi(y(s)) y(s)=0
\end{aligned}
$$

or

$$
\begin{aligned}
0 \geqq & {\left[\Gamma_{0}(s)(y(s) \phi(y(s)))^{-1}\right]_{T_{1}}^{t}-\int_{T_{1}}^{t} \Gamma_{0}(s) D\left((y(s) \phi(y(s)))^{-1}\right) d s } \\
& -M\left|N_{1} N_{2}\right| \int_{T_{1}}^{t} \frac{s^{2 k-1} y_{2 k}(s)}{y(s) \phi(y(s))} d s \\
& +\int_{T_{1}}^{t} s^{2 n} y_{\tau}(s) f\left[s, y_{\tau}(s)\right] d s / y(s) \phi(y(s)) .
\end{aligned}
$$

Since $\phi>0, \phi^{\prime} \geqq 0$ and $\phi^{\prime \prime} \leqq 0, \phi^{\prime}$ is a positive decreasing function on $\left[T_{0}, \infty\right)$ so that

$$
\phi(t)-\phi\left(T_{1}\right)=\int_{T_{1}}^{t} \phi^{\prime}(s) d s \geqq\left(t-T_{1}\right) \phi^{\prime}(t),
$$

that is.

$$
\left(t-T_{1}\right) \phi^{\prime}(t) \leqq \phi(t)-\phi\left(T_{1}\right)<\phi(t)
$$

for $t \geqq T_{1}$. Thus, $t \phi^{\prime}(t) \leqq \phi(t)$ for $t \geqq 2 T_{1}$. Either of these inequalities implies that $\lim _{t \rightarrow \infty} \phi^{\prime}(t) / \phi(t)=0$. We consider

$$
\left|\frac{\phi\left(y_{\tau}(t)\right)}{\phi(y(t))}-1\right|=\frac{\phi(y(t))-\phi\left(y_{\tau}(t)\right)}{\phi(y(t))}=\frac{\tau(t) \phi^{\prime}(\mu)}{\phi(y)}
$$

for some $\mu$, where $y_{\tau}(t)<\mu<y(t)$. Since $\phi$ is an increasing function, $\phi\left(y_{\tau}(t)\right)<\phi(\mu)<\phi(y(t))$, which implies that $1 / \phi(y(t))<1 / \phi\left(y_{\tau}(t)\right)$. Similarly, $\phi^{\prime}$ is a decreasing function so that $\phi^{\prime}(\mu)<\phi^{\prime}\left(y_{\tau}(t)\right)$. Thus, $\phi^{\prime}(\mu) / \phi(y(t))<\phi^{\prime}\left(y_{\tau}(t)\right) / \phi\left(y_{\tau}(t)\right)$. Moreover, because $k \geqq 1, \lim _{t \rightarrow x} y_{\tau}(t)=\infty$, which shows that

$$
\lim _{t \rightarrow \infty} \frac{\phi\left(y_{\tau}(t)\right)}{\phi(y(t))}=1 .
$$

Thus, for any $\varepsilon$ with $0<\varepsilon<1$, there is a $t_{\varepsilon} \geqq T_{1}$ such that

$$
\phi\left(y_{r}(t)\right) \geqq(1-\varepsilon) \phi(y(t)), \quad t \geqq t_{\text {f. }}
$$


By Lemma 2, there is a $k_{0}>0$ and a $t_{0} \geqq T_{1}$ such that $y_{\tau}(t) \geqq k_{0} y(t)$ for $t \geqq t_{0}$. Thus, for $s \geqq T=\max \left\{t_{0}, t_{e}, 2 T_{1}\right\}$

$$
\begin{aligned}
s^{2 n} \frac{y \tau(s)}{y(s)} \frac{f\left[s, y_{\tau}(s)\right]}{\phi(y(s))} & \geqq k_{0}(1-\varepsilon) s^{2 n} \frac{f\left[s, y_{\tau}(s)\right]}{\phi(y(s))} \\
& \geqq k_{0}(1-\varepsilon) s^{2 n} f\left(s, C s^{j}\right) .
\end{aligned}
$$

By Lemma 1 and a change of variables

$$
\begin{gathered}
\int_{T_{1}}^{t} \frac{s^{2 k-1} y_{2 k}(s)}{y(s) \phi(y(s))} d s \geqq N_{2 k} \int_{T_{1}}^{l} \frac{y^{\prime}(s) d s}{y(s) \phi(y(s))} \\
N_{2 k} \int_{y\left(T_{1}\right)}^{y(t)} \frac{d u}{u \phi(u)} .
\end{gathered}
$$

We may now duplicate the rest of the arguments of the proof of Theorem 2 to obtain the following result.

Corollary 1. Let $f$ be nonlinear with strength coefficient $2 n+1-j$. Let the associated function $\phi$ satisfy $\phi^{\prime \prime}(u)<0$. Then (2) fulfills property $P_{0}$ if for all positive constants $C$

$$
\int^{\infty} t^{2 n} f\left(t, C t^{j}\right) d t=\infty .
$$

REMARK 1. If $\tau \equiv 0$, the ratio $y_{\tau}(t) / y(t)$ does not occur and the condition $\phi^{\prime \prime}(u)<0$ may be omitted.

Remark 2. When $r \equiv 1, \tau \equiv 0$ and $k \equiv 0$, Theorem 1 reduces to the sufficiency of Theorem 1 of Kiguradze (1962). Under the same conditions the conclusion of Theorem 2 is that (2) satisfies property $P_{0}$, that is, condition $A$; Theorem 2 then coincides with Theorem 3 of Kiguradze (1962). In view of Remark 1, when $r \equiv 1, \tau \equiv 0$ and $j=0$, Theorem 3 reduces to the sufficiency of Theorem 5 of Kiguradze (1962).

Remark 3. When $r \equiv 1, \tau \equiv 0, k=n$, the conclusion of Theorem 2 is that any $C_{n}$-solution $y(t)$ of (2) must satisfy $\lim _{t \rightarrow \infty} y_{2 n}(t)=0$. This is not quite condition B since we have not shown that any positive solution of (2) has this property. If $r(t) \equiv 1$, property $P_{n}$ reduces to condition $B$. For suppose that $y(t)$ is any solution of (2) of type $C_{k}$, where $k<n$, then $D^{2 n-1} y(t)=y_{2 n-1}(t)<0$. We now invoke a lemma most recently stated in generalized form by Ladas (1971) which we adapt here as follows.

Lemma 3. Let $y$ be a positive solution of (2) on $\left[t_{0}, \infty\right)$ with $r \equiv 1$. Then

$$
\lim _{t \rightarrow \infty} D^{2 n} y(t)=\lim _{t \rightarrow \infty}(j-1) !\left(t-t_{0}\right)^{t-j} D^{2 n+1-j} y(t)
$$

where $j=1, \cdots, 2 n+1$. 
Letting $j=2$, we note that $D^{2 n} y(t)>0$ so that $\lim _{t \rightarrow \infty} D^{2 n} y(t) \geqq 0$. On the other hand, $D^{2 n-1} y(t)<0$, which implies that the limit on the right-hand side is nonpositive and $\lim _{t \rightarrow \infty} D^{2 n} y(t)=0$.

When $r(t) \not \equiv 1$, the statement of this lemma is more complicated. We may, however, state a weak analogue.

Lemma $3^{\prime}$. Let $y(t)$ be a positive solution of (2) on $\left[t_{0}, \infty\right)$ with $j \geqq$ $2 n+1-i$. Then

$$
\lim _{t \rightarrow \infty} y_{2 n}(t)=\lim _{t \rightarrow \infty}(j-1) !\left(t-t_{0}\right)^{1-j} y_{2 n+1-j}(t),
$$

where $j=1, \cdots, 2 n+1-i$.

Now let $y$ be a $C_{k}$-solution of (2) on $\left[T_{0}, \infty\right)$ for $k=0, \cdots, n-1$. Then $y_{2 n-1}(t)<0$ for $t \geqq T_{0}$. We see then that if $i \leqq 2 n-1,2 n-i \geqq 1$ and $2 n-i+1 \geqq 2$. Thus, we may let $j=2$ in the statement of Lemma $3^{\prime}$ to obtain as before that $\lim _{t \rightarrow \infty} y_{2 n}(t)=0$. It follows that if $i \leqq 2 n-1$, the conclusion of Theorem 2 may be restated as: (2) fulfills condition B. Specifically, property $P_{n}$ and condition $B$ coincide for the equation

$$
D^{n+1}\left[r(t) D^{n} y(t)\right]+y_{\tau}(t) f\left[t, y_{\tau}(t)\right]=0, \quad n \geqq 1
$$

since $i=n \leqq 2 n-1$ if $n \geqq 1$. The same remark holds for

$$
D^{n}\left[r(t) D^{n+1} y(t)\right]+y_{\tau}(t) f\left[t, y_{\tau}(t)\right]=0, \quad n \geqq 2
$$

since $i=n+1 \leqq 2 n-1$ if $n \geqq 2$.

REMARK 4. Use of the preliminary transformation $Y(t)=-y(t)$ will enable us to formulate criteria for the nonexistence of negative solutions $y$ of (2) for which $-y$ is of type $C_{k}(k=0, \cdots, n)$. Moreover, if (2) has property $P_{k}$, then there are no negative solutions $y$ such that $-y$ is of type $C_{r}$ $(r=k+1, \cdots, n)$ and any negative solution $y$ for which $-y$ is of type $C_{k}$ will satisfy $\lim _{t \rightarrow \infty} y_{2 k}(t)=0$.

3

In this section we consider the nonhomogeneous delay differential equation

$$
D^{2 n+1-i}\left[r(t) D^{i} y(t)\right]+y_{\tau}(t) f\left[t, y_{\tau}(t)\right]=Q(t) .
$$

Following the procedure introduced and used most effectively by Kartsatos and Manougian (to appear), we shall assume that $R$ is a solution of the ordinary differential equation

$$
D^{2 n+1-i}\left[r(t) D^{i} R(t)\right]=Q(t) .
$$


This permits the transformation of (18) to a homogeneous delay equation of order $2 n+1$ for which the methods of the previous sections may be applied. Let us assume that $y$ is a positive solution of (18) and let $u(t)=y(t)-R(t)$. Then

$$
\begin{aligned}
D^{2 n+1-i}\left[r(t) D^{i} u(t)\right] & =D^{2 n+1-i}\left[r(t) D^{i} y(t)\right]-D^{2 n+1-i}\left[r(t) D^{i} R(t)\right] \\
& =-y_{\tau}(t) f\left[t, y_{\tau}(t)\right]=-(u+R)_{\tau}(t) f\left[t,(u+R)_{\tau}(t)\right],
\end{aligned}
$$

so that $u$ is a solution of the homogeneous equation

$$
D^{2 n+1-i}\left[r(t) D^{i} u(t)\right]+(u+R)_{\tau}(t) f\left[t,(u+R)_{\tau}(t)\right]=0 .
$$

Since $y(t)>0$ for $t \geqq T_{0},(u+R)_{\tau}(t)>0$ for $t \geqq T_{1}$ and $D^{2 n+1-i}\left[r(t) D^{i} u(t)\right]<0$ for $t \geqq T_{1}$, which implies that $u(t)$ is a nonoscillatory solution of (20). If $u(t)<0$, then we further transform the equation by letting $v(t)=-u(t)$. It follows that $v$ is a positive solution of

$$
D^{2 n+1-i}\left[r(t) D^{i} v(t)\right]-(R-v)_{\tau}(t) f\left[t,(R-v)_{\tau}(t)\right]=0 .
$$

Definition 8. A positive solution $y$ of $(18)$ is of type $C_{k}^{R}$ on $\left[T_{0}, \infty\right)$ for $k=0, \cdots, n$ if $u=y-R$ is a positive solution of (20) of type $C_{k}$ on $\left[T_{0}, \infty\right)$.

Definition 9. A positive solution y of (18) is of type $\hat{C}_{k}^{R}$ on $\left[T_{0}, \infty\right)$ for $k=0, \cdots, n-1$ if $v=R-y$ is a positive solution of (21) which for $t \geqq T_{0}$ satisfies

$$
v_{i}(t)>0, i=0, \cdots, 2 k+1 \text { and }(-1)^{i+1} v_{i}(t)>0, i=2 k+2, \cdots, 2 n .
$$

It is of type $\hat{C}_{n}^{R}$ if $v_{i}(t)>0$ for $i=0, \cdots, 2 n$.

Definition 10. Equation (18) has property $P_{k}^{R}$ if, and only if, (20) has property $P_{k}$.

A positive solution of (18) is evidently of type $C_{k}^{R}(k=0, \cdots, n)$ or of type $\hat{C}_{k}^{R}(k=0, \cdots, n)$ for some $k$. We now formulate criteria under which (18) possesses property $P_{k}^{R}$.

THeOrem 3. Let $R$ be a solution of (19). Any solution y of type $C_{k}^{R}$ will satisfy

$$
\lim _{t \rightarrow \infty}[y(t)-R(t)]_{2 k}=0
$$

if for all positive constants $C$

$$
\int^{\infty} t^{2 n-2 k}\left(R_{\tau}(t)+C t^{2 k}\right) f\left[t, R_{\tau}(t)+C t^{2 k}\right] d t=\infty .
$$

Proof. Let $y$ be a solution of (18) of type $C_{k}^{R}$ on $\left[T_{0}, \infty\right)$. As in Theorem 1, we obtain 


$$
\int^{t} s^{2 n-2 k} D y_{2 n}(s) d s+\int^{t} s^{2 n-2 k}(u+R)_{\tau}(s) f\left[s,(u+R)_{\tau}(s)\right] d s=0 .
$$

The first integral is handled as in Theorem 1 . It remains to estimate the second integral. Since $u$ is of type $C_{k}$ on $\left[T_{0}, x\right)$, there are numbers $k_{0}>0, N_{2 k}>0$, $C>0$ such that

$$
u_{\tau}(t) \geqq k_{0} u(t) \geqq k_{0} N_{2 k}^{-1} t^{2 k} u_{2 k}(t) \geqq k_{0} N_{2 k}^{-1} C t^{2 k}
$$

provided we assume that $\lim _{t \rightarrow x} u_{2 k}(t)=C>0$. The above inequalities will lead to the same contradiction as in the proof of Theorem 1.

TheOREM 4. Suppose that $R$ is as in the hypothesis of Theorem 3 and that $\phi$ is a function satisfying $\phi(y)>0, \phi^{\prime}(y) \geqq 0$ and $(9 a)$. Equation (18) possesses property $P_{k}^{R}$ if in addition to (23) for all positive constants $C$

$$
\int^{x} t^{2 n} f\left(t, R_{\tau}(t)+C t^{2 k}\right) s t / \phi(t)=x .
$$

Proof. As in the proof of Theorem 2, we first show that

$$
\int^{\infty} t^{2 n} f\left(t, R_{r}(t)+C t^{2 k-1}\right) d t / \phi(t)=\infty, \quad k \geqq 1
$$

is sufficient to exclude solutions of type $C^{R}(s=k, \cdots, n)$. Then

$$
\int^{\infty} t^{2 n} f\left(t, R_{\tau}(t)+C t^{2 k+1}\right) d t / \phi(t)=x, \quad k \geqq 0
$$

and hence (24) will exclude solutions of (18) of type $C_{s}^{R}(s=k+1, \cdots, n)$. The details are left to the reader.

REMARK 5 . If $R(t)>0$, the condition of Theorem 3 may be replaced by

$$
\int^{\infty} t^{2 n} f\left(t, C t^{2 k}\right) d t=\infty
$$

The same replacement in Theorem 4 may be made. Thus (25) will ensure that (18) has property $P_{k}^{R}$.

REMARK 6. If $R$ is oscillatory or negative, there can be no solutions of (18) of type $\hat{C}_{k}^{R}$. The conclusion of Theorem 4 is thereby strengthened.

REMARK 7. Use of the transformation $w_{k}(t)=y_{2 n}(t) y_{2 k-1}^{-1}(t)$ results in a stronger criterion for the nonexistence of $C_{k}{ }_{k}$-solutions of (18) independent of the existence of an auxiliary function $\phi(t)$ satisfying the hypotheses of Theorem 4. Specifically, we may obtain:

THEOREM 5. Let $R$ be a solution of (19) with $R(t)=0\left(t^{2 k-1-t}\right)$ for some $\varepsilon$ such that $0<\varepsilon<2 k-1$; (18) has no positive solutions of type $C_{s}^{R}$ ( $s=$ $k, \cdots, n)$ if for all positive constants $C$ 


$$
\int^{\infty} t^{2 k-1} f\left(t, R_{\tau}(t)+C t^{2 k-1}\right) d t=\infty
$$

REMARK 8. As in Remark 4, the preliminary transformation $Y(t)=-y(t)$ will enable us to formulate analogous criteria for the nonexistence of certain negative solutions $y$ of (18) for which $-y$ is a positive $C_{k}^{-R}$-solution of the transformed non-homogeneous delay differential equation.

\section{References}

A. G. Kartsatos and M. N. Manougian (to appear), 'Perturbations causing oscillations of functional differential equations', Proc. Amer. Math. Soc.

I. T. Kiguradze (1962), 'Oscillation properties of solutions of certain ordinary differential equations', Dokl. Akad. Nauk SSR. 144, 33-36.

G. Ladas (1971), 'On principal solutions of nonlinear differential equations', J. Math. Anal. Appl. 36, 103-109.

R. D. Terry (1974), 'Oscillatory properties of a delay differential equation of even order', Pacific $J$. Math. 52, 269-282.

R. D. Terry (1973), 'Oscillatory properties of a fourth-order delay differential equation, 2', Funkcial. Ekvac. 16, 213-224.

California Polytechnic State University

San Luis Obispo

California 93407

U.S.A. 